\title{
Khan Academy: as potencialidades como objeto de aprendizagem
}

\author{
Khan Academy: the potential as an learning object
}

Passiano Scott Puhl ${ }^{1}$
Pontifícia Universidade Católica do Rio Grande do Sul (PUCRS), Porto Alegre, RS, Brasil
(D) https://orcid.org/0000-0003-0696-5666, 9 http://lattes.cnpq.br/1383741674721811

Bruno Resende ${ }^{2}$

Pontifícia Universidade Católica do Rio Grande do Sul (PUCRS), Porto Alegre, RS, Brasil (D) https://orcid.org/0000-0003-0528-2222, 9 http://lattes.cnpq.br/4225420227314950

\section{Thaísa Jacintho Müller ${ }^{3}$ \\ Pontifícia Universidade Católica do Rio Grande do Sul (PUCRS), Porto Alegre, RS, Brasil (D) https://orcid.org/0000-0002-7986-202X, 9 http://lattes.cnpq.br/9841564835844236}

\begin{abstract}
Resumo: Este artigo apresenta uma avaliação de um ambiente da plataforma Khan Academy como um objeto de aprendizagem (OA), verificando as suas potencialidades para a compreensão do conteúdo de ângulos formados por retas paralelas cortadas por uma transversal. $\mathrm{Na}$ avaliação do $\mathrm{OA}$ foram considerados os seguintes critérios: usabilidade, interação, interatividade, acessibilidade, qualidade do conteúdo, efetividade e apresentação. A análise foi predominantemente qualitativa, enfatizando-se a descrição ao invés dos dados numéricos. Concluiu-se que o OA possui potencial para proporcionar a compreensão do conteúdo de ângulos formados por retas paralelas cortadas por uma transversal, visto que a plataforma tem um ambiente agradável e motivador para que o estudante se envolva na realização das atividades, bem como na organização didática dos conteúdos, proporcionando que o estudante aprenda no seu ritmo.
\end{abstract}

Palavras-chave: Plataforma Khan Academy; Avaliação; Ensino de Matemática.

Abstract: This article presents an evaluation of a Khan Academy platform environment as a learning object (LO), verifying its potential for understanding the content of angles formed by parallel lines cut by a transversal one. The evaluation was carried out with an LO assessment instrument, in which the following criteria were considered: usability, interaction, interactivity, accessibility, content quality, effectiveness and presentation. The analysis was predominantly qualitative, emphasizing the description instead of the numerical data. It was concluded that the LO has the potential to provide an understanding of the content of angles formed by parallel straight lines cut by a cross, since the platform has a pleasant and motivating environment for the student to be involved in carrying out the activities, as well as in the didactic display of contents, allowing students to learn at their own pace.

Keywords: Khan Academy Platform; Evaluation; Mathematics Teaching.

Data de submissão: 30 de novembro de 2020.

Data de aprovação: 10 de agosto de 2021.

${ }^{1}$ Currículo sucinto: Doutor em Educação em Ciências e Matemática pela Pontifícia Universidade Católica do Rio Grande do Sul (2021). Mestre em Ensino de Ciência e Matemática pela Universidade de Caxias do Sul (2016). Graduado em Licenciatura em Matemática pela Universidade de Caxias do Sul (2012). Professor da rede municipal de Bom Princípio, Rio Grande do Sul, Brasil. Contribuição de autoria: Análise Formal, Escrita - Primeira Redação, Escrita - Revisão e Edição, Investigação. Contato: c.s.puhl@hotmail.com.

${ }^{2}$ Currículo sucinto: Mestre em Educação em Ciências e Matemática pela Pontifícia Universidade Católica do Rio Grande do Sul (PUCRS). Graduado em Licenciatura em Matemática pela Universidade Federal do Rio Grande do Sul (UFRGS). Contribuição de autoria: Análise Formal, Escrita - Primeira Redação, Escrita - Revisão e Edição. Contato: bruno.resende@acad.pucrs.br.

${ }^{3}$ Currículo sucinto: Doutora em Informática na Educação pela Universidade Federal do Rio Grande do Sul (2015). Mestre em Matemática pela Universidade Federal do Rio Grande do Sul (2010). Graduada em Licenciatura Plena em Matemática pela Pontifícia Universidade Católica do Rio Grande do Sul (2007). Professora da Pontifícia Universidade Católica do Rio Grande do Sul (PUCRS). Contribuição de autoria: Escrita - Revisão e Edição. Contato: thaisamuller@gmail.com. 


\section{Introdução}

O avanço tecnológico transformou e vem transformando a cultura, a sociedade e a função social da escola. Dessas instituições, a escola é a que apresenta maior resistência em transformar-se e adequar-se às necessidades da Sociedade da Informação ou da Sociedade em Rede (BEHAR, 2009). Na Sociedade Industrial do século XIX, a função social da escola era propiciar aos estudantes o acesso às informações e aos conhecimentos construídos durante o avanço da sociedade, preparando-os para desempenhar papéis de acordo com suas aptidões e habilidades, ou seja, o objetivo central da escola estava no processo de ensino (MORETTO, 2007; BEHAR, 2009).

No século XXI, a evolução das tecnologias, principalmente com o advento da web, inicia uma revolução na Educação, pois ocorre a dinamização, a divulgação e o fácil acesso à informação e ao conhecimento (MORETTO, 2007). Desse modo, não cabe à escola privilegiar uma prática mecânica, no processo de ensino, mas organizar-se para promover a aprendizagem (KENSKI, 2006; BEHAR, 2009). Diante disso, a escola não pode se limitar ao acesso aos conteúdos disciplinares, mas precisa promover a compreensão dos conteúdos aos estudantes, além de desenvolver competências e habilidades, o respeito mútuo, a colaboração, a cooperação, a solidariedade, a autonomia, o respeito ao ritmo individual, entre outras (MORAN; MASETTO; BEHRENS, 2002; KENSKI, 2006; BEHAR, 2009).

Contudo, observa-se que a escola ainda está distante de atingir esses objetivos. As Tecnologias Digitais de Informação e Comunicação (TDIC) podem auxiliar e agilizar a adaptação da escola para as necessidades da Sociedade em Rede. Entre os vários recursos digitais disponíveis no Brasil, destaca-se o estudo e a produção de objetos de aprendizagem (OA). Se construído adequadamente, seguindo alguns critérios didáticos e gráficos, torna-se um recurso eficaz para apresentar conhecimentos de forma mais dinâmica e interativa, além de permitir ao estudante determinar o ritmo da realização das atividades e da aprendizagem.

A plataforma Khan Academy ${ }^{1}$, em suas unidades de aprendizagem, pode ser considerada um $\mathrm{OA}$, constituído basicamente de vídeo-aulas e exercícios disponibilizados gratuitamente. Essa plataforma é um projeto educacional, desenvolvido pelo norte-americano, Salman Kahn, cujo objetivo, é "[...] oferecer ensino de classe mundial, de graça, a qualquer pessoa, em qualquer lugar" (FUNDAÇÃO LEMANN, 2018). Na perspectiva de Marc Prensky (2011) e Gary Stager (THOMPSON, 2011), essa plataforma está longe de revolucionar os processos de ensino e de

\footnotetext{
1 "A Khan Academy oferece exercícios, vídeos de instrução e um painel de aprendizado personalizado que habilita os estudantes a aprender no seu próprio ritmo dentro e fora da sala de aula. [...] Nossas missões de matemática guiam os estudantes do jardim de infância até o cálculo, usando tecnologias adaptativas de ponta que identificam os pontos fortes e lacunas no aprendizado" (FUNDAÇÃO LEMANN, 2018). A plataforma pode ser acessada em: https://pt.khanacademy.org/brasil.
} 
aprendizagem, mas é uma ótima iniciativa para inserir as tecnologias na escola, além de promover uma educação de qualidade a todos de forma gratuita.

Diante desse contexto, decidiu-se fazer uma investigação sobre essa plataforma, tendo o seguinte problema norteador: "Quais são as potencialidades da plataforma Khan Academy, como OA, para se ensinar ângulos formados por retas paralelas cortadas por uma transversal?" Em busca de respostas a esse questionário, realizou-se uma avaliação da plataforma Khan Academy seguindo alguns parâmetros como: usabilidade, interação, interatividade, acessibilidade, qualidade do conteúdo, efetividade e apresentação. Desse modo, este tem o objetivo de verificar as potencialidades da plataforma Khan Academy para a aprendizagem de conhecimentos sobre os ângulos formados por retas paralelas cortadas por uma transversal.

O artigo está organizado em quatro seções. A primeira aborda elementos do referencial teórico, definindo um $\mathrm{OA}$ e as suas características. Já na segunda seção apresentam-se os procedimentos metodológicos, sendo que o ponto central são as decisões metodológicas assumidas na execução do estudo. A terceira seção apresenta os resultados da experiência vivenciada com estudantes do oitavo ano do Ensino Fundamental, de uma escola pública do interior do Rio Grande do Sul, e avaliação realizada da plataforma como um OA. Por fim, são expostas as conclusões, com as possíveis respostas ao problema norteador.

\section{Referencial teórico}

A utilização das TDIC em processos de ensino e de aprendizagem não é garantia de sucesso, porém muitos recursos, como os objetos de aprendizagem (OA), buscam qualificar esses processos. Os OA surgiram com o objetivo de facilitar a localização de recursos digitais na web, podendo ser reutilizados em diferentes contextos e em diferentes ambientes de aprendizagem (WILEY, 2000; BEHAR, 2009; SINGO, 2014). Os OA, geralmente, são armazenados em repositórios digitais, tais como: Banco Internacional de Objetos Educacionais ${ }^{2}$, Recursos Educacionais Abertos de Matemática ${ }^{3}$, Khan Academy, entre outros. Apesar desses objetivos, os OA ainda não possuem uma definição conceitual única empregada pela comunidade científica.

Neste trabalho optou-se por defini-lo como "[...] qualquer recurso digital que possa ser reutilizado para dar suporte à aprendizagem" (WILEY, 2000, p. 3). Nesse contexto, os OA são pequenas entidades digitais que podem ser utilizadas, e reutilizadas, simultaneamente por várias pessoas em diferentes contextos de aprendizagem (WILEY, 2000). Por essa definição, um OA pode ser qualquer material digital, como, por exemplo, textos, animação, vídeos, imagens, aplicações, páginas web de forma isolada ou em combinação, desde que propiciem a

2 http://objetoseducacionais.mec.gov.br/\#/inicio

${ }^{3}$ https://oerworldmap.org/resource/urn:uuid:6b811921-a325-403b-8488-9ff4afa3386c?language=pt 
compreensão dos conteúdos (BEHAR, 2009). Neste caso, o OA utilizado consiste de um ambiente da plataforma Khan Academy.

Contudo, conforme já foi ressaltado, somente a utilização das TDIC, ou mais especificamente de $O A$, não necessariamente qualificam os processos de ensino e de aprendizagem, mas estes recursos possuem características diferenciadas para proporcionar aprendizagens, se comparados com os métodos tradicionais (SOUZA JÚNIOR; LOPES, 2007; BEHAR, 2009; SANTAROSA, 2010; SINGO, 2014). A utilização de OA possibilita processos de ensino e de aprendizagem apoiado pela tecnologia, no qual o professor pode mudar a função de transmissor de conhecimento para a de mediador (BEHAR, 2009).

Apesar de não existir um consenso, a princípio, há características em comum em todos os OA: reusabilidade, portabilidade, modularidade, metadados e interatividade (TAROUCO, 2004). A reusabilidade refere-se às pequenas entidades digitais que podem ser utilizadas em múltiplos contextos e ambientes de aprendizagem, além de adequar-se com diferentes estratégias didáticas. A portabilidade consiste na flexibilidade do OA ser executado em diferentes plataformas de trabalho (sistemas operacionais). Outra característica que dinamiza e qualifica o OA é a modularidade, sendo compreendido como entidades digitais independentes e não sequenciais que permitem a utilização em múltiplos contextos e com outros recursos. Em busca de facilitar a organização e localização de OA, há os metadados, que fornecem diferentes informações aos usuários, como: título, autor, descrição, objetivos, características que mostram como, quando e por quem o objeto foi desenvolvido, armazenado, entre outros. E por último, a característica mais importante, a interatividade, que se refere ao nível de interação entre o conteúdo do OA com o estudante. Quanto maior a interação propiciada, mais qualificado é o OA, pois, possivelmente, substituirá um estudante passivo por um sujeito ativo nos processos de ensino e de aprendizagem (BEHAR, 2009).

Diante desse contexto, a construção de um OA não é fácil (NASCIMENTO, 2007), sendo necessários conhecimentos em diferentes áreas para desenvolver materiais interativos, em relação ao uso da tecnologia e com objetivos didáticos claros e definidos para o planejamento pedagógico desses materiais. Desse modo, ao criar um OA faz-se pertinente uma equipe interdisciplinar de professores e técnicos, para desenvolvê-lo considerando-se aspectos técnicos, estéticos, pedagógicos e motivacionais. Especificamente, em relação aos aspectos pedagógicos, os OA podem ser predominantemente instrutivos ou mais interativos, dependendo do modelo pedagógico que conduziu a sua criação (BEHAR, 2009; SANTAROSA, 2010).

No atual cenário educacional, devem-se evitar estratégias expositivas ou meramente instrutivas, mas buscar o desenvolvimento de OA interacionistas para permitir a compreensão de conteúdos e a formação integral e crítica do estudante, ao contrário do instrucionista que enfatiza a memorização, o treinamento e o reforço (SILVA; FERNANDEZ, 2007; BEHAR, 2009). 
Complementando essa perspectiva, os OA aplicados em Matemática possuem potencial para "[...] o desenvolvimento do pensamento lógico e do espírito investigativo, através da resolução de situações-problemas, que servirão para compreender e transformar sua realidade" (SANTAROSA, 2010, p. 286).

Por fim, além do processo de criação de OA faz-se necessário realizar uma avaliação. Há diferentes instrumentos de avaliação de OA, neste trabalho utilizou-se um modelo proposto por Lieban et al. (2018) ${ }^{4}$, avaliando as seguintes características: usabilidade, interação, interatividade, acessibilidade, qualidade de conteúdo, efetividade e apresentação.

A usabilidade é entendida como a "medida na qual um produto pode ser usado por usuários específicos para alcançar objetivos específicos com eficácia, eficiência e satisfação em um contexto específico de uso" (ABNT, 2002, p. 3). Neste trabalho, a usabilidade está relacionada com a facilidade de o estudante compreender o funcionamento e a navegação do OA (BEHAR, 2009).

Interação é definida como "[...] uma influência mútua de organismos inter-relacionados; ação mútua ou compartilhada entre dois ou mais corpos ou indivíduos; comunicação e relações entre pessoas que convivem" (INTERAÇÃO, 2009). O OA busca envolver o estudante no processo de aprendizagem, por meio de diferentes recursos, promovendo a interatividade, sendo assim a interatividade é definida como "[...] um tipo de relação tecno-social, ou seja, como um diálogo entre homem e máquina, por meio de interfaces gráficas, em tempo real" (BEHAR, 2009, p. 51). Segundo Lieban et al. (2018), a interatividade é um mecanismo necessário e fundamental para a compreensão de conteúdos e o desenvolvimento de habilidades cognitivas e físicas.

A acessibilidade é compreendida como o acesso do usuário "[...] independentemente de suas capacidades físico-motoras e perceptivas, culturais e sociais, usufruir os benefícios de uma vida em sociedade, ou seja, é a possibilidade de participar de todas as atividades, [...] com o mínimo de restrições possível" (OLIVEIRA JÚNIOR; FERREIRA, 2010). Considerando essa definição e o contexto da pesquisa, a acessibilidade está relacionada na organização do OA, para que possa ser utilizado também por estudantes que tenham alguma limitação físico-motora.

Em relação aos demais critérios, Lieban et al. (2018) define qualidade do conteúdo como "[...] um critério que envolve o rigor conceitual, a profundidade do conteúdo e a condizência com o tema proposto"; efetivação, "[...] diz respeito à capacidade que o OA tem de promover resultados pretendidos"; e apresentação é "[...] referente ao projeto visual do OA e aos componentes estéticos da interface". Essas são as definições dos critérios adotados para realizar avaliação da plataforma Khan Academy como OA. A seguir descreve-se sobre o objeto de aprendizagem utilizado e o instrumento de avaliação adotado.

\footnotetext{
${ }^{4} \mathrm{O}$ instrumento de avaliação está disponível em: https://www.dropbox.com/s/xomhw84casfhpek/protocolo final2.xlsx?dl=0. Acesso em: 8 abr. 2020.
} 


\section{Descrição do objeto de aprendizagem e do instrumento de avaliação}

O OA avaliado, pelos autores desta pesquisa, consiste em um ambiente da plataforma Khan Academy, cujo conteúdo que é abordado refere-se a ângulos ${ }^{5}$ - especificamente sobre a relação entre os ângulos formados por retas paralelas e transversais -, com estudantes do $8^{\circ}$ ano do Ensino Fundamental. A escolha desse conteúdo justifica-se pela dificuldade que os estudantes têm em compreender a relação de equivalência entre os ângulos formados por retas paralelas e transversais. Esse fato está baseado na experiência docente dos presentes autores. Diante disso, o OA oportuniza que o estudante aprenda no seu ritmo: abstraindo as informações, visualizando os ângulos, analisando a situação e concluindo sobre a equivalência dos ângulos.

\section{A plataforma Khan Academy}

[...] oferece exercícios, vídeos de instrução e um painel de aprendizado personalizado que habilita os estudantes a aprender no seu próprio ritmo dentro e fora da sala de aula. Abordamos matemática, ciência, programação de computadores, história, história da arte, economia e muito mais. Nossas missões de matemática guiam os estudantes do jardim de infância até o cálculo, usando tecnologias adaptativas de ponta que identificam os pontos fortes e lacunas no aprendizado. Também temos parcerias com instituições como a NASA, o Museu de Arte Moderna, a Academia de Ciências da Califórnia e o MIT para oferecer conteúdo especializado (FUNDAÇÃO LEMANN, 2018).

Essa plataforma tem o objetivo de oferecer uma estrutura gratuita de recursos educacionais, buscando fornecer aulas de qualidade para estudantes de todas as idades. Para acessar todos os recursos disponíveis na plataforma faz-se necessário realizar um cadastro no site ou no aplicativo disponível para smartphone conectando-se por meio de uma conta do Facebook ou do Google.

Nessa plataforma, os estudantes assistem vídeos sobre um conteúdo específico para posteriormente realizar exercícios para verificar o nível de compreensão atingido. Os professores podem criar um ambiente personalizado, uma sala de aula virtual, com o objetivo de ajudar na aprendizagem dos seus estudantes, sugerindo exercícios, vídeos, artigos, além de acompanhar o desempenho dos mesmos. Nesta sala de aula o professor acompanha o desenvolvimento individual dos estudantes, verifica os conteúdos e o tempo dedicado ao estudo, bem como os conhecimentos em que estão apresentando maiores dificuldades. Para os pais, a plataforma oferece a possibilidade de gerenciar uma conta para os filhos com menos de 12 anos de idade, tendo a finalidade de fornecer informações aos pais sobre o desenvolvimento dos exercícios online realizados por seus filhos.

Neste trabalho, o OA selecionado está estruturado conforme uma sala de aula tradicional, na qual realiza-se uma exposição do conteúdo, por meio de uma vídeo-aula, para, posteriormente, realizar exercícios para verificar o nível de compreensão do estudante. No entanto, por se tratar

\footnotetext{
${ }^{5}$ Este é o link do OA que será descrito e avaliado: https://pt.khanacademy.org/math/geometry/hs-geofoundations/hs-geo-angles/v/measuring-angles-in-degrees.
} 
de OA, o estudante não precisa seguir necessariamente a ordem dos conteúdos, ou ainda assistir a aula para depois fazer os exercícios. Caso o estudante tenha convicção ou queira testar seu nível de conhecimento, há a possibilidade de ir direto para os exercícios sobre o conteúdo, sem assistir a vídeo aula.

Por exemplo, na Figura 1 o painel à esquerda lista em ordem de complexidade alguns conteúdos relacionados a ângulos, sendo que o primeiro vídeo mostra como utilizar o transferidor para medir ângulos. Os estudantes do $8^{\circ}$ ano do Ensino Fundamental, geralmente, já manusearam o transferidor e mediram ângulos, assim há a possibilidade de não assistir os vídeos informativos (Medição de ângulos em graus; Como medir ângulos usando um transferidor; Como medir ângulos usando um transferidor 2), realizando direto uma lista de exercícios (Praticar: meça ângulos).

Figura 1 - Painel que dispõe dos conteúdos organizados por nível de dificuldade

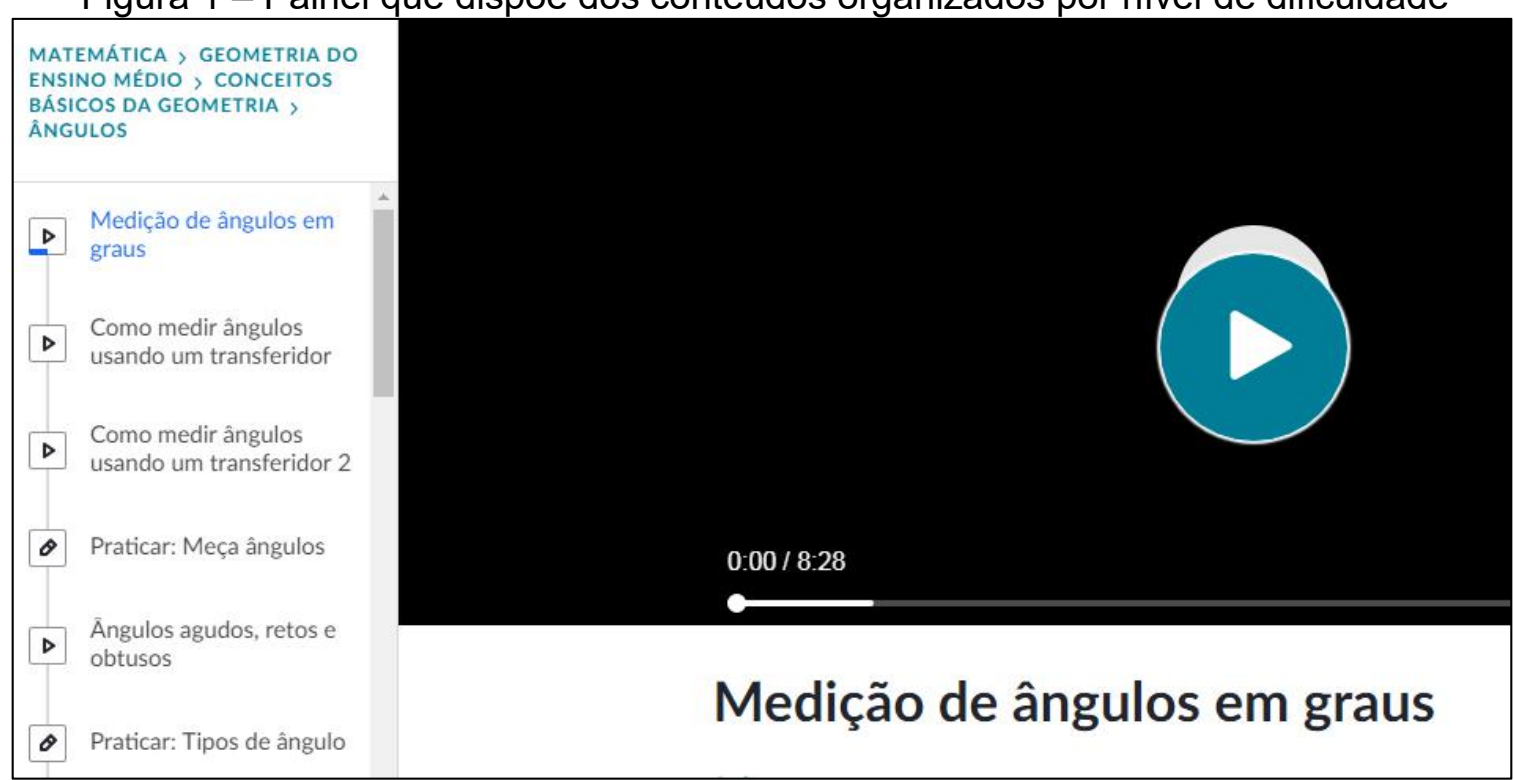

Fonte: Captura de tela da plataforma Khan Academy.

Ao final de cada lista de exercícios é disponibilizado um resumo do aproveitamento do estudante, em percentual do número de acertos que obteve (Figura 2). Caso o estudante tenha encontrado dificuldades, a plataforma disponibiliza um texto e um vídeo explicativo sobre o conteúdo, sendo esse um recurso para realizar uma revisão do que foi estudado. 
Figura 2 - Resumo dos exercícios de medir os ângulos

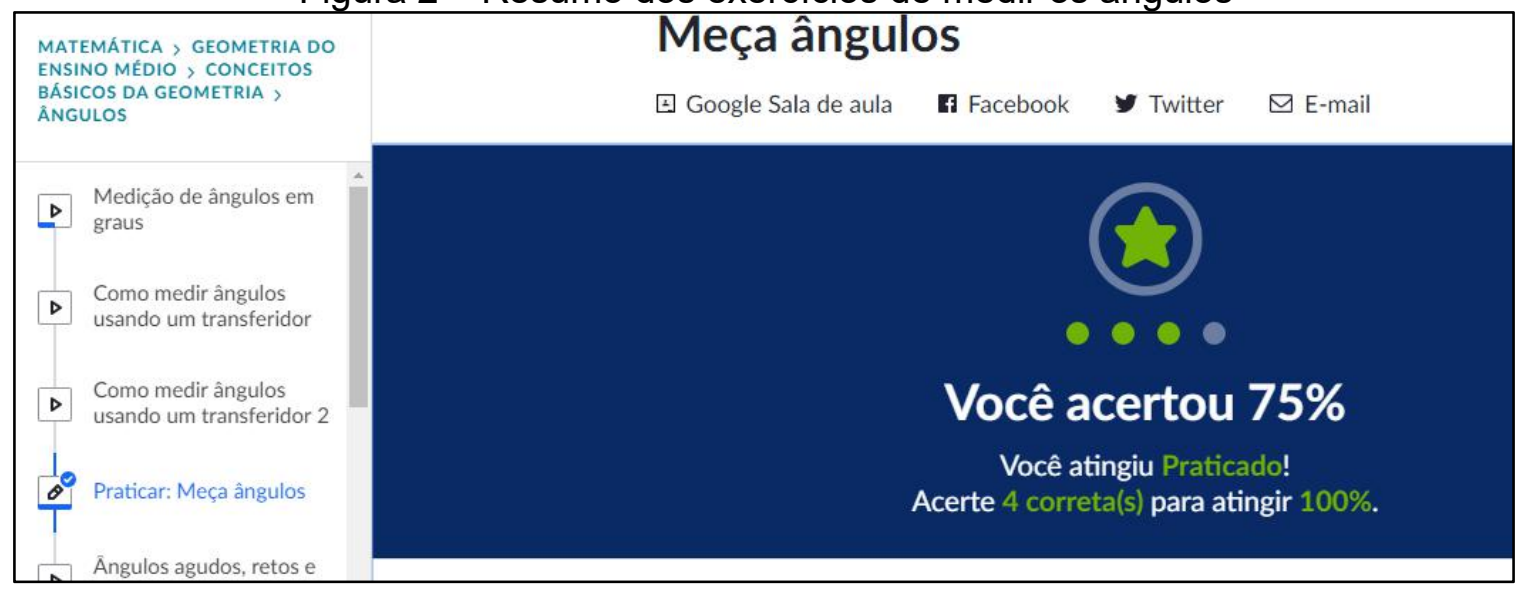

Fonte: Captura de tela da plataforma Khan Academy.

Além disso, ao realizar os exercícios, os estudantes não precisam utilizar caderno e lápis, pois na plataforma há um recurso que permite a escrita dos procedimentos de cálculo, bem como há a possibilidade de usar uma calculadora para os exercícios considerados mais complicados (Figura 3). Porém, o recurso da escrita e da calculadora não podem ser utilizados simultaneamente, pois ocorrem em ambientes diferentes.

Figura 3 - Recursos da Khan Academy para auxiliar na resolução do exercício

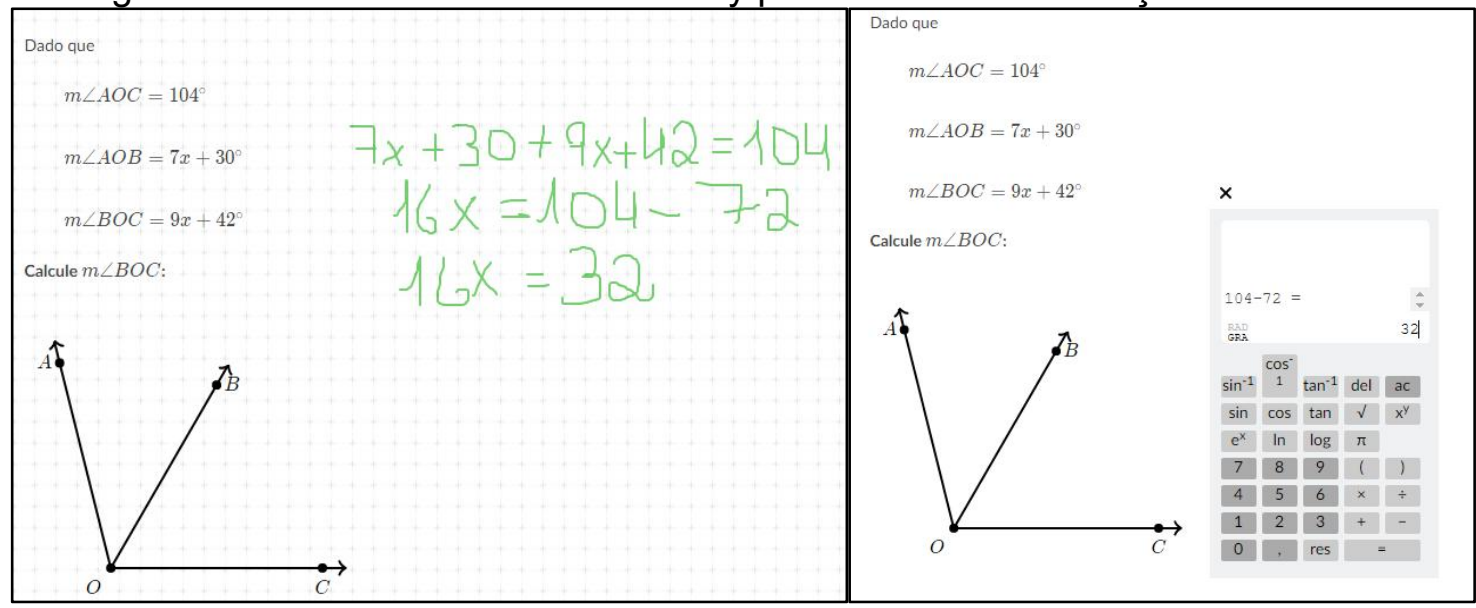

Fonte: Captura de tela da plataforma Khan Academy.

Apesar da plataforma ter uma semelhança com uma sala de aula tradicional, de uma aula expositiva, há aspectos que a difere, pois, os estudantes ao interagir com OA podem pausar o vídeo, rever explicações, refazer exercícios a quantidade de vezes que for necessário, permitindo com que cada estudante compreenda o conteúdo no seu ritmo. Sendo assim, a plataforma Khan Academy busca proporcionar uma aprendizagem autônoma, sem a necessidade de ter um professor explicando ou corrigindo os exercícios.

A aplicação da plataforma Khan Academy ocorreu durante o segundo semestre de 2018, sendo utilizada por uma turma de oitavo ano do Ensino Fundamental de uma escola pública no interior do Rio Grande do Sul. O professor, que é um dos autores do artigo, disponibilizou o link do 
ambiente aos estudantes e acompanhou-os na interação com OA, observando as limitações e as potencialidades desse recurso didático para o processo de aprendizagem do conteúdo de ângulos formados por retas paralelas cortadas por uma transversal. Além da observação, o professor avaliou a plataforma Khan Academy como OA, para isso utilizou-se um instrumento proposto por Lieban et al. (2018) que avalia os seguintes critérios: usabilidade, interação, interatividade, acessibilidade, qualidade do conteúdo, efetividade e apresentação.

Além do professor, de forma independente, os demais autores dessa pesquisa também avaliaram o OA. O instrumento de avaliação de Lieban et al. (2018) é composto de perguntas, cujas respostas consistem na seguinte escala: insatisfatório; satisfatório; bom; muito bom; e ótimo. Cada escala corresponde a uma porcentagem do critério avaliado: insatisfatório, $0 \%$; satisfatório, $25 \%$; bom, 50\%; muito bom, $75 \%$; ótimo, 100\%; e não sei, desconsidera a resposta. Quanto mais alto o percentual obtido, mais o $\mathrm{OA}$ atende àquela característica. Ao final, é mostrado um gráfico de barras, cada uma correspondendo a um critério. Sendo assim, é possível, visualmente, ter uma noção da qualidade do objeto que se está buscando ou trabalhando.

Contudo, neste estudo, além de se utilizar o modelo de Lieban et al. (2018), em cada critério apresentou-se justificativas para a escala atribuída. Apesar de ter sido utilizado um modelo que proporciona dados numéricos, a análise é predominante qualitativa, pois busca-se avaliar e descrever as potencialidades do OA. A avaliação foi realizada a partir da interatividade dos estudantes com os recursos do OA.

A interatividade com $\mathrm{OA}$ ocorreu no laboratório de informática na escola, no qual o professor sugeriu esse recurso para os estudantes iniciarem os estudos sobre ângulos. Os estudantes acessaram livremente o OA, seguindo as atividades sugeridas. Inicialmente, o professor não realizou nenhuma intervenção com os estudantes, em relação a explicações dos conteúdos abordado na plataforma Khan Academy.

Durante a interatividade com OA, observou-se que os estudantes estavam empenhados em compreender o conteúdo abordado, seja em texto ou em vídeo, e em realizar as atividades as propostas. Os estudantes estavam atentos nas explicações, principalmente nos recursos audiovisuais e interativos. Porém, quando eram disponibilizados exercícios, os estudantes apresentavam dificuldades em resolvê-los, principalmente nos exercícios que envolviam equações e equivalência de ângulos, como, por exemplo: ângulos opostos pelo vértice, ângulos suplementares e ângulos complementares.

Os estudantes buscaram superar as dificuldades solicitando auxílio do professor e resolvendo as equações no seu caderno. Apesar dessas dificuldades, o OA cumpriu seu objetivo de promover a compressão de conceitos introdutórios do conteúdo de ângulos formados por retas paralelas cortadas por uma transversal. A seguir apresenta-se a avaliação realizada do OA. 


\section{Análise dos resultados}

Em relação à usabilidade, os autores dessa pesquisa classificaram o OA analisado como "muito bom" (Figura 4) por apresentar uma estrutura que facilita o compartilhamento de diferentes conteúdos, cursos e contextos de áreas distintas e, principalmente, por fazer parte de uma organização maior dentro dos conteúdos matemáticos. Além disso, conforme o estudante demonstra a compressão dos conteúdos, o OA, junto ao professor, pode adaptar e disponibilizar diferentes atividades, contemplando as defasagens de aprendizagem. Entretanto, o OA é um ambiente fechado que não permite o acréscimo de materiais didáticos ou qualquer tipo de alteração nas atividades do OA. Apesar de ser um ambiente fechado, a plataforma permite com que o professor indique conteúdos e atividades para os estudantes, conforme as dificuldades individuais, promovendo um ensino individualizado.

Figura 4 - Avaliação do critério da usabilidade

\begin{tabular}{|c|c|c|c|c|c|c|}
\hline USABILIDADE & INSATISFATÓRIO & SATISFATÓRIO & вом & МUIто вом & о́тімо & não sei opinar \\
\hline $\begin{array}{l}\text { O OA apresenta condição de ser compartilhado entre } \\
\text { diferentes cursos ou contextos de aprendizagem sem } \\
\text { modificação? }\end{array}$ & & & & $X$ & & \\
\hline $\begin{array}{l}\text { O OA é passivel de adaptação ou realimentação em } \\
\text { função de necessidades diagnosticadas no processo? }\end{array}$ & & & & $\mathrm{X}$ & & \\
\hline O OA permite seu uso em diversos cursos e contextos? & & & & $X$ & & \\
\hline $\begin{array}{l}\text { O OA é parte de um conjunto maior, ou seja, está } \\
\text { associado a outros objetos de aprendizagem? }\end{array}$ & & & & X & & \\
\hline
\end{tabular}

Fonte: Elaborado pelos autores baseado na avaliação realizada sobre o Khan Academy.

Em relação à interação, os autores dessa pesquisa o avaliaram como "bom" (Figura 5), pois no decorrer das atividades existe a possibilidade do professor ou de estudantes fornecerem feedbacks e dicas para qualificar os processos de ensino e de aprendizagem. Porém, esse aspecto poderia ser aperfeiçoado, criando-se um ambiente especial com tópicos das principais dúvidas, facilitando e promovendo uma maior interação.

Figura 5 - Avaliação do critério da interação

\begin{tabular}{|c|c|c|c|c|c|c|}
\hline INTERAÇÃO & INSATISFATÓRIO & SATISFATÓRIO & BOM & MUITо вом & о́тімо & não sei opinar \\
\hline $\begin{array}{l}\text { No OA há chat, fóruns ou outros recursos que proporcionem trocas } \\
\text { sincronas ou assincronas? }\end{array}$ & & & $x$ & & & \\
\hline
\end{tabular}

Fonte: Elaborado pelos autores baseado na avaliação realizada sobre o Khan Academy.

Os autores dessa pesquisa consideraram a interatividade como "bom" (Figura 6), pois não há muitas opções de interatividade. O estudante pode manipular, com o mouse, um transferidor para medir ângulos, uma caneta para fazer registros de cálculos e uma calculadora. Esses são exemplos de interatividade utilizados na execução de atividades de resolução de exercícios. 
Figura 6 - Avaliação do critério da interatividade

\begin{tabular}{|c|c|c|c|c|c|c|}
\hline $50 \%$ & INSATISFATÓRIO & SATISFATÓRIO & BOM & MUITO вом & о́тімо & nâo sei opinar \\
\hline $\begin{array}{l}\text { O OA apresenta um direcionamento que incite o aluno à } \\
\text { manipulação de seus elementos? }\end{array}$ & & & $X$ & & & \\
\hline $\begin{array}{l}\text { Os componentes "clicáveis" contribuem para a } \\
\text { construção do conhecimento? }\end{array}$ & & & $X$ & & & \\
\hline
\end{tabular}

Fonte: Elaborado pelos autores baseado na avaliação realizada sobre o Khan Academy.

A qualidade do conteúdo inserida no OA foi caracterizada pelos autores dessa pesquisa como "ótimo". O conteúdo dos vídeos e da plataforma são contextualizados, com os conceitos explanados de forma clara e de fácil compreensão. As sequências dos vídeos são coerentes e com abordagens visualmente atrativas. Além disso, cada vídeo apresenta pequenos fragmentos dos conteúdos, de modo que os vídeos não são extensos, mas ao mesmo tempo apresentam com detalhes os principais conceitos. Outro aspecto que potencializa a aprendizagem é a combinação de textos, imagens e animações, permitindo diferentes maneiras do estudante compreender o conteúdo. Independente do recurso, as atividades possuem instruções claras e estão em conformidade com o nível de escolaridade proposto aos estudantes pelo OA.

Figura 7 - Avaliação do critério da qualidade do conteúdo

\begin{tabular}{|c|c|c|c|c|c|c|}
\hline QUALIDADE DO CONTEÚDO & INSATISFATÓRIO & SATISFATÓRIO & BOM & MUITO BOM & Ótıмо & não sei opinar \\
\hline Quanto à veridicidade, o OA é... & & & & & $X$ & \\
\hline O conteúdo do OA é claro e conciso? & & & & & $\mathrm{X}$ & \\
\hline Há precisão na apresentação das informações? & & & & & $\mathrm{X}$ & \\
\hline $\begin{array}{l}\text { Há adequação do nível e do detalhamento das } \\
\text { informações apresentadas? }\end{array}$ & & & & & $X$ & \\
\hline O OA apresenta bem o conceito? & & & & & $X$ & \\
\hline $\begin{array}{l}\text { O O aborda o nível de ensino, curso(s) e características } \\
\text { do aluno? }\end{array}$ & & & & & $\mathrm{X}$ & \\
\hline $\begin{array}{l}\text { As atividades e avaliações propostas são condizentes aos } \\
\text { objetivos propostos pelo OA? }\end{array}$ & & & & $x$ & & \\
\hline $\begin{array}{l}\text { O nível de complexidade é adequado ao público alvo } \\
\text { pensado? }\end{array}$ & & & & $X$ & & \\
\hline
\end{tabular}

Fonte: Elaborado pelos autores baseado na avaliação realizada sobre o Khan Academy.

Contudo, como esta é uma plataforma desenvolvida nos Estados Unidos, encontrou-se alguns erros de tradução, conforme é observado na Figura 8. O ângulo reto foi traduzido como "direita" e o ângulo raso como "linha reta". Esse erro de tradução pode atrapalhar o estudante, ocasionando um erro na questão por uma tradução incorreta. 
Figura 8 - Erro de tradução de algumas palavras no OA

O ângulo a seguir é agudo, reto, obtuso ou raso?

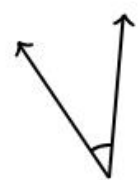

Escolha 1 resposta:

Acutângulo

Direita

Obtusângulo

Em linha reta

Fonte: Captura de tela da plataforma Khan Academy.

A acessibilidade foi considerada pelos presentes autores como "ótimo" (Figura 9), pois o manuseio da plataforma é intuitivo e possui um design prático e de fácil acesso aos estudantes, permitindo trocas rápidas dos ambientes de aprendizagem. Além disso, o OA pode ser acessado por diferentes sistemas operacionais, tanto no computador como nos smartphones. Contudo, o único aspecto da acessibilidade não contemplado refere-se à utilização da plataforma por estudantes com deficiência, assim entende-se que este é um aspecto que o OA precisa qualificarse, para que este recurso digital também possa ser aproveitado por estes estudantes.

Figura 9 - Avaliação do critério da acessibilidade

\begin{tabular}{|l|l|l|l|l|l|l|}
\hline ACESSIBILIDADE & \multicolumn{1}{c|}{ MOM ÓITO BOM } \\
\hline No OA há suporte a alunos com necessidades especiais?
\end{tabular}

Fonte: Elaborado pelos autores baseado na avaliação realizada sobre o Khan Academy.

Sobre a efetividade, os presentes autores classificaram como "muito bom" (Figura 10), pois os conhecimentos que serão estudados são explicitados e os vídeos estão organizados hierarquicamente, em que os conceitos gerais são abordados antes dos específicos. Essa organização dos conteúdos proporciona uma compreensão gradativa dos conhecimentos pelos estudantes, partindo dos conhecimentos gerais para a compreensão de conceitos mais específicos. Sendo assim, ressalta a importância de relacionar os conhecimentos para que o estudante possa compreender o conteúdo e não simplesmente realizar uma aprendizagem mecânica. 
Figura 10 - Avaliação do critério da efetividade

\begin{tabular}{||l|l|l|l|l|l|l||}
\hline EFEIIVIDADE ÓtIMO & não sei opinar \\
\hline Explicita os objetivos de aprendizagem? & & & $X$ & & \\
\hline Apresenta os conceitos progressiva e claramente? & & & & $X$ & \\
\hline Apresenta conceitos prévios necessários para sua utilização? & & & & $X$ & \\
\hline Demonstra relações entre conceitos? & & & & $X$ & & \\
\hline Favorece a aprendizagem em menor tempo? & & & $X$ & & \\
\hline
\end{tabular}

Fonte: Elaborado pelos autores baseado na avaliação realizada sobre o Khan Academy.

Em relação ao critério da apresentação do OA, classificou-se como "ótimo" (Figura 11), pois possui um design atrativo e busca promover a ação cognitiva do estudante, motivando-o a resolver as atividades, a ganhar uma pontuação maior. Além disso, não se observa uma sobrecarga cognitiva, em que as cores são utilizadas para despertar a atenção dos estudantes.

Figura 11 - Avaliação do critério da apresentação

\begin{tabular}{|c|c|c|c|c|c|c|}
\hline APRESENTACCÃO & INSATISFATÓRIO & SATISFATÓRIO & Bом & MUIто вом & б́тімо & não sei opinar \\
\hline É visualmente atraente? Tem projeto visual aprimorado? & & & & & $x$ & \\
\hline Solicita dos alunos, na sua execução, operações mentais eficientes? & & & & & $x$ & \\
\hline $\begin{array}{l}\text { Tem potencial para engajar, motivar e despertar o interesse e } \\
\text { curiosidade dos alunos? }\end{array}$ & & & & & $x$ & \\
\hline
\end{tabular}

Fonte: Elaborado pelos autores baseado na avaliação realizada sobre o Khan Academy.

A avaliação de cada critério está sintetizada na Figura 12, apresentando fortes indícios de que este OA possui potencial para ser utilizado em sala de aula, permitindo a compreensão do conteúdo pelos estudantes por meio dos vídeos e das atividades propostas. Entretanto, considerase que o mesmo pode ser aperfeiçoado em alguns aspectos, como permitir que o recurso da caneta e da calculadora sejam utilizados de forma simultânea, corrigir os erros de tradução e principalmente tomar uma atenção especial para o OA, para ser utilizado por pessoas com deficiência.

Figura 12 - Gráfico geral da avaliação do OA

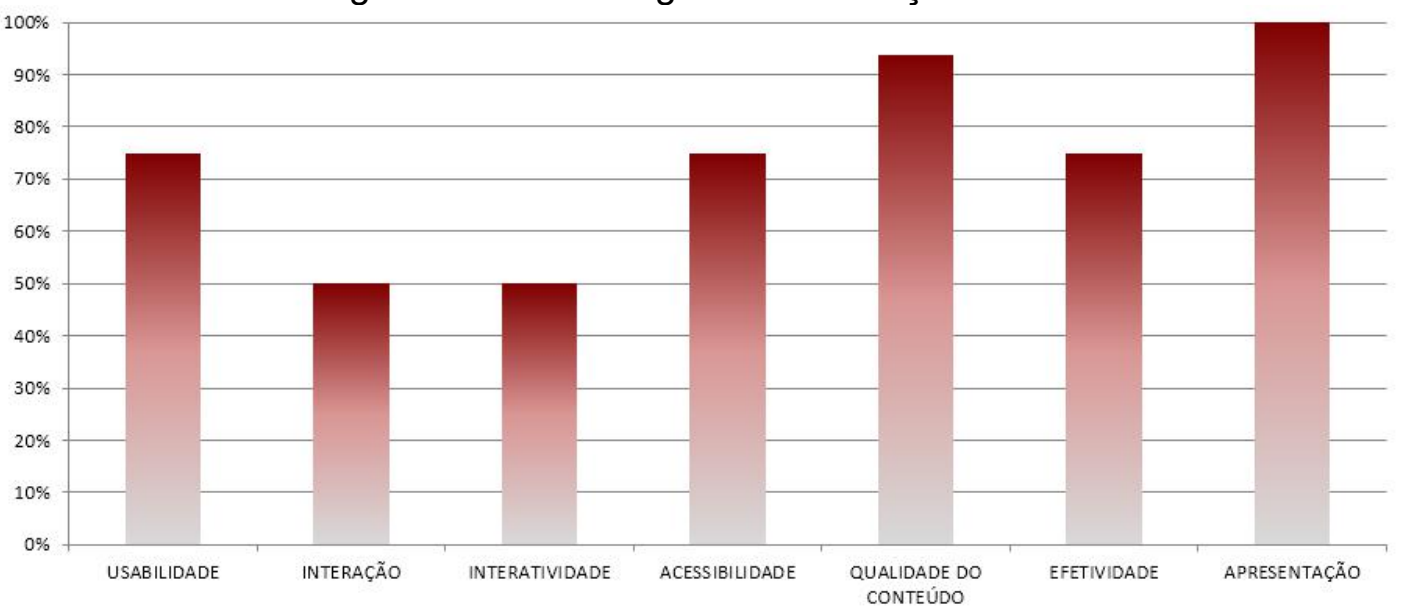

Fonte: Elaborado pelos autores baseado na avaliação realizada sobre o Khan Academy.

Por fim, por meio das descrições e das avaliações realizadas entende-se que o OA possui potencial para qualificar os processos de ensino e de aprendizagem, podendo atingir o seu 
objetivo em sala de aula. O OA permite que os estudantes compreendam o conteúdo exposto nos vídeos: as relações envolvidas no estudo dos ângulos formados por retas paralelas e transversais.

\section{Considerações finais}

A plataforma Khan Academy vem ganhando novos usuários a cada dia, tornando-se um recurso utilizado por estudantes em todos os níveis de escolaridade, para aprenderem ou revisarem conteúdos que não possuem domínio, ou por professores para inovar nas suas práticas em sala de aula. Em virtude disso, decidiu-se analisar uma parte da plataforma, como um OA para o ensino do conteúdo de ângulos formados por retas paralelas cortadas por um transversal, buscando verificar as potencialidades da plataforma Khan Academy como um OA.

A organização do conteúdo da plataforma não difere dos livros didáticos e do ensino expositivo presente em sala de aula. Os vídeos são de caráter informativo e os exercícios buscam reforçar os conhecimentos expostos no vídeo, em um nível gradativo de dificuldade. Contudo, diferente da sala de aula, na plataforma pode-se parar as explicações, realizar diversas vezes as atividades, ou seja, as atividades são realizadas e os estudantes podem aprender no seu ritmo.

Diante disso, cabe destacar a eficiência da plataforma em ter um ambiente agradável e motivador para que o estudante se envolva na realização das atividades, bem como na organização didática dos conteúdos. A plataforma está organizada para privilegiar os conhecimentos prévios para, posteriormente, abordar conceitos mais específicos, estabelecendo a relação entre esses conhecimentos e permitindo a compreensão de novos conteúdos. Se o estudante estiver motivado e predisposto em realizar as atividades, possivelmente, compreenderá os conteúdos estudados. Além dessas potencialidades aos estudantes, o professor, ao criar uma sala de aula virtual, pode realizar uma avaliação formativa, pois a plataforma proporciona indicativos do nível de conhecimento do estudante bem como as áreas que possuem defasagem e o tempo dedicado em cada atividade da plataforma.

Portanto, este OA pode ser utilizado por estudantes - para aprendizagens autônomas por professores - como atividades em sala de aula -, sendo um exemplo de tecnologia que é aliada na qualificação dos processos de ensino e de aprendizagem. A plataforma precisa de aperfeiçoamentos para realizar o sonho do seu idealizador, Salman Kahn, em proporcionar um ensino de qualidade de graça a todos no mundo. Apesar da necessidade das melhorias, este OA já possui potencial para proporcionar a compreensão dos conteúdos, sem a intervenção de um professor, desde que o estudante esteja motivado e envolvido nas atividades. Por fim, destaca-se que, atualmente, os diferencias da plataforma Khan Academy são de proporcionar diferentes ritmos e trajetórias de aprendizagem, adaptando as atividades conforme os conhecimentos de cada estudante, além de proporcionar ao professor uma avaliação formativa individual, mostrando indicativos do nível de conhecimento de cada estudante. 


\section{Referências}

ABNT. Associação Brasileira de Normas Técnicas. NBR 9241-11: Requisitos Ergonômicos para Trabalho de Escritórios com Computadores. Parte 11: Orientações sobre Usabilidade. Rio de Janeiro: ABNT, ago. 2002.

BEHAR, Patricia Alejandra. Modelos pedagógicos em educação a distância. Porto Alegre: Artmed, 2009.

FUNDAÇÃO LEMANN. Quem Somos. 2018. Disponível em: https://fundacaolemann.org.br/somos\#somos-vision. Acesso em: 13 nov. 2018.

INTERAÇÃO. In: HOUAISS, A. Dicionário Eletrônico Houaiss da Língua Portuguesa. Rio de Janeiro: Objetiva, 2009.

KENSKI, Vani Moreira. Tecnologias e ensino presencial e a distância. 3. ed. Campinas, SP: Papirus, 2006.

LIEBAN, Diego Eduardo; JABOINSKI, Lilian de Cássia Nunes; MÜLLER, Thaísa Jacintho; FANTINEL, Patrícia da Conceição; DAUDT, Sônia Isabel Dondonis. Avaliação de Objetos de Aprendizagem. Disponível em: http://oaquatromaisum.wix.com/oa. Acesso em: 5 dez. 2018.

MORAN, José Manuel; MASETTO, Marcos T.; BEHRENS, Marilda Aparecida. Novas tecnologias e mediação pedagógica. Campinas, SP: Papirus, 2002.

MORETTO, Vasco Pedro. Prova: um momento privilegiado de estudo, não um acerto de contas. Rio de Janeiro: Lamparina, 2007.

NASCIMENTO, Anna Christina de Azevedo. Objetos de Aprendizagem: a distância entre a promessa e a realidade. In: PRATA, Carmem Lúcia; NASCIMENTO, Anna Christina de Azevedo. Objetos de Aprendizagem: Uma Proposta de Recurso Didático. Brasília: MEC, SEED, 2007.

OLIVEIRA JÚNIOR, Jorge Fiore de; FERREIRA, Simone Bacellar Leal. Guia de Referência em Acessibilidade Web. Rio de Janeiro: UNIRIO, 2010. Disponível em: http://acessibilidadelegal.com/13-guia.php. Acesso em: 15 mar. 2020.

PRENSKY, Marc. Khan Academy. Educational Technology, v. 51, n. 5, jul./ago. 2011.

SANTAROSA, Lucila Maria Costi (Org.). Tecnologias digitais acessíveis. Porto Alegre: JSM Comunicação Ltda., 2010.

SILVA, Rejane Maria G.; FERNANDEZ, Márcia Aparecida. Recursos informáticos projetados para o ensino de Ciências: bases epistemológicas implicadas na construção e desenvolvimento de objetos de aprendizagem. In: PRATA, Carmem Lúcia; NASCIMENTO, Anna Christina de Azevedo. Objetos de Aprendizagem: Uma Proposta de Recurso Didático. Brasília: MEC, SEED, 2007.

SINGO, Felix. Objetos de aprendizagem multimodais. In: LIMA, José Valdeni; SINGO, Felix; CANTO FILHO, Alberto; MÜLLER, Thaísa; SILVA, Flávia (Org.). Objetos de aprendizagem multimodais: projetos e aplicações. Barcelona: Editorial UOC, 2014.

SOUZA JÚNIOR, Arlindo José de; LOPES, Carlos Roberto. Saberes docentes e o desenvolvimento de objetos de aprendizagem. In: PRATA, Carmem Lúcia; NASCIMENTO, Anna 
Christina de Azevedo. Objetos de Aprendizagem: Uma Proposta de Recurso Didático. Brasília: MEC, SEED, 2007.

TAROUCO, Liane Margarida Rockenbach. Avaliação de objetos de aprendizagem. 2004.

Disponível em: http://penta2.ufrgs.br/edu/objetosaprendizagem. Acesso em: 2 jul. 2018.

THOMPSON, Clive. How Khan Academy is changing the rules of education. Wired Magazine, v. 126, 15 jul. 2011.

WILEY, David. The instructional use of learning objects. Online Version. 2000. Disponível em http://reusability.org/read. Acesso em: 20 out. 2018. 